\title{
A Discrete-Time Convergence Model for Proliferation-able Stem Cell and its Estimation using Kalman Filter
}

Obaidul Malek $^{1 *}$, Anastasios Venetsanopoulos ${ }^{1}$, Laila Alamgir ${ }^{2}$, Javad Alirezaie ${ }^{1}$ and Sridhar Krishnan ${ }^{1}$

${ }^{1}$ Electrical and Computer Engineering, Ryerson University, Toronto, ON, Canada

${ }^{2}$ Department of Medicine, Howard University, Washington DC, USA

\begin{abstract}
The main challenge of stem cell biology is to characterize proliferation and differentiation processes; since, very little is known regarding the molecular stimuli responsible for their regulatory mechanisms. On the other hand, comprehensive molecular analysis is yet too complex to perform intuitively. Therefore, computational models are essential for the optimization of clinical understanding in order to enhance the therapeutic process.

In this article, a discrete-time convergence model for stem cell growth process based on clinical observations and engineering predictions has been proposed. Typically, stem cell populations are in quiescent; but in response to molecular stimuli they become activated and proliferate, and undergo divisional cycle before experiencing the terminal differentiation or disappearance process. The objective of this paper is to present a computational analysis of stem cell proliferation process, and standardize a model which converges with the experimental hypothesis. More importantly, it has also been shown that, inherent homogeneous and heterogeneous properties of stem cell populations are also the necessary conditions for this convergent theory. In addition, Kalman filter has been used for estimating the unknowns as well as effciency of the proposed model. Simulation results based on synthetic data are presented to illustrate the performance of the proposed technique.
\end{abstract}

Keywords: Convergence; Proliferation-able stem cell; Kalman filter; Computational model; Stochastic process

\section{Introduction}

Stem cells need little conceptual background as proliferation and differentiation processes which are responsible for repairing and replacing damage tissues are still undefined. Clinical studies found that after receiving the molecular stimuli such as biological injuries, stem cells undergo activation, proliferation and differentiation processes for repopulating the injured area in order to compensate the losses [1]. Even, in the unperturbed situation, damage to proliferation-able cell during transition from the proliferation to differentiation stage also needs to be compensated for the sake of continuation of this (cyclic) process $[1,2]$. However, this process is invariably heterogeneous, and the capacity as well as potential of this process is largely dependent on the cell types. Furthermore, cells from the surrounding subpopulation are also responsible for this regeneration process; and given their unique regenerative dynamics, stem cells improve the capability to treat debilitating diseases like cancer, diabetes, heart and muscular dystrophy.

Multicellular organisms in human cells have inherent ability to replicate under its respective regulatory constraints in order to meet the requirements of a normal healing process $[2,3]$. According to the clinical studies, cells can be categorized in several subclasses including quiescent cells, stem cells, progenitor cells, terminally differentiated cells and dead cells (apoptosis). In this present context, stem cell is considered as proliferation-able as long as it has the capability of self-renewal or the property of growing through the symmetrical or asymmetrical cell division process [4-9]. In contrast, differentiated cells may or may not have the cell division potential through the symmetrical or asymmetrical cell division, while dead cell is the disappearance of the cell from the cell life cycle. Conceptually, the biology of cell proliferation, differentiation and apoptosis in cell culture is remarkably similar for all cell types; however, overall process can be influenced by their respective unique regulatory and environmental factors.

Fundamentally, proliferation is the process which increases the cell volume or mass. It is a time variant, heterogeneous and random process; where, even some differentiated cells have the probability to proliferate and increases the cell population (cell quantity) by symmetric and asymmetric cell division processes; while others lose that potential. Furthermore, rate of proliferation is extremely dependent on the number of initial cell populations, rate of cell division, growth factors and their proliferative capability $[2,3]$. However, cell populations lose these properties when they reach at the end of their life cycle known as terminal differentiation or disappearance (cell death or apoptosis) [3]. Differentiation, differential gene expression-a change in the set of genes, starts when the growing cells follow the fertilization and undergoes phenotypic and morphological changes to a complex tissue specific cell types. This process is relatively slow and partially or completely ceases the cell's ability to further proliferate (terminal differentiation), and may also be responsible to perform specialized function for a long period of time. While, apoptosis, cell death or disappearance, is a necessary mechanism in order to ensure homeostasis of all tissues. It is basically a complement of growth process, which needs to be highly regulated because of the extended cell survival. This is the coordinated function of tissue and morphogenesis, which is responsible to create a permissible platform in case of genetic instability and accumulation of mutations.

The main focal point of this proposed quantification and estimation processes is to design a discrete engineering architecture for the

*Corresponding author: Obaidul Malek, Electrical and Computer Engineering, Ryerson University, Toronto, ON, Canada, E-mail: omalek03@gmail.com

Received December 17, 2012; Accepted January 17, 2013; Published January 22, 2013

Citation: Malek O, Venetsanopoulos A, Alamgir L, Alirezaie J, Krishnan S (2013) A Discrete-Time Convergence Model for Proliferation-able Stem Cell and its Estimation using Kalman Filter. J Bioengineer \& Biomedical Sci 3: 119. doi:10.4172/2155-9538.1000119

Copyright: ( 2013 Malek O, et al. This is an open-access article distributed under the terms of the Creative Commons Attribution License, which permits unrestricted use, distribution, and reproduction in any medium, provided the original author and source are credited. 
proliferative cell populations which converges with the experimental hypothesis. Furthermore, the formulation of this model is based on the observations of cell growth, differentiation and apoptosis in the cell culture media (i.e., in vitro) [2]. The goal of this paper is also to generalize a novel discrete time convergence computational theory, which would be able to execute and analyze the characteristics of stem cell populations in temporal domain. More importantly, the foundation of this generalization has been compiled under the assumption that proliferation, differentiation and apoptosis processes are exceedingly similar for all cell types. However, they are influenced by the respective cell properties. On the other hand, cell populations under study exhibits inherent heterogeneous property as a whole and homogeneous during their transition process. In addition, they have finite probability and completed their life cycle through the terminally differentiation or disappearance process. The main biomedical challenge of this paper is to model the parameters which are responsible for the stimulation of the respective cell properties including the unique regulatory, environmental, permissive and repressive factors. Other challenges associated with this research are the integration of the cell biology with the engineering paradigms as well as finding the biological resources and previous work hypothesis.

Furthermore, muscle stem cell also known as satellite cell has been used for the model study. Satellite cells are small cell populations, initially identified in 1961, residing in indentations between the sarcolemma and the basement membrane [10]. The regeneration process of skeletal muscle following a physiological demand is largely dependent on muscle stem cells. Studies also found that, this cell population has remarkable capability to adapt with the physiological injuries including growth and training [1]. Typically, muscle stem cell populations are quiescent and remain in nonproliferationable and nondifferentiation-able states. However, in response to mechanical stimuli such as myotrauma, muscle stem cells become activated and proliferate as muscle myoblasts, also known as myogenic markers, before undergoing myogenic differentiations (or terminally differentiations) for augmenting the existing muscle fibers or forming new fibers [11]. This process is invariably heterogeneous due to the diverse nature of intracellular interactions [12]. Clinicians and biologists are trying to understand the dynamics and complexities of their behaviors based on the clinical and experimental observations. Despite of many researches, generalization of these regulatory factors as well as the overall understanding of how these function to contribute the sequential events in the muscular development are still unclear. In addition, scope for the intuitive analysis of its nature is very complex and infeasible. Therefore, computational model becomes a crucial part for satellite cell biology.

Motivation of this paper is its comprehensive quantitative analysis of proliferation-able stem cell populations and its convergence theory; however, this phenomenon can also be implementable for cell differentiation process. More importantly, the execution of the computational process is based on the detection, prediction and estimation theory and a Kalman filter has been used to formulate this analytical architecture as well as evaluation of its performance. In addition, stem cell biology is a promising area of clinical inquiry where most of the fundamental concepts are still undefined [1]. Therefore, this paradigm could be used as a new predicted microscope for the researchers to investigate the quantitative theory in order to characterize the growth process; since, almost none of the computational models have yet been developed.

Remainder of the paper is organized as follows. Section 2 studies fundamentals of computational theory for stem cell architecture.
Section 3 presents formulation of computational model, quantitative analysis, transition properties and convergence of the model. Section 4 carries out an estimation technique using Kalman filter. Simulations and result are presented in Section 5. Finally, conclusions are included in Section 6.

\section{Computational Cell Architecture}

Satellite cells are a pole of quiescent and undifferentiated mononucleated myogenic cells, located at the periphery of terminally differentiated and multinucleated muscle fibers. Like other stem cells, these tissue specific progenitor cells have the remarkable proliferation and self-renewal capabilities. However, these cell populations are transcriptionally inactive, but activate and express molecular markers of the MyoD or myf5 families in response to injuries [4] and play a vital role in the maintenance and the regeneration of skeletal muscle. Furthermore, satellite cells not only have remarkable ability for muscle regeneration and remodeling processes, but also play vital roles in the muscular therapeutic process in order to treat the deadly diseases such as cachexia or muscular dystrophy. In addition, myosatellite cell numbers and its distribution between the muscle groups are dependent on the age and type of the muscle fibers [5]. Studies found that the molecular phenotypes responsible for these processes could express distinct developmental commitment due to the heterogeneity in the satellite cell contents, and because of this property even one tissue vary in their fundamental cell types [2]. A complete life cycle of the satellite cell following a myotrauma is presented in figure 1.

The in vitro experiment for satellite cell is designed to analyze the proliferation-able cell populations in temporal and spatial domains. Proposed model has assumed three types of cell populations: stem cell (or progenitor cells) with the finite proliferation-able or self-renewal capability; differentiated cells, which have very limited proliferative capability and lose their propagative properties through terminal differentiation process; and dead cells, which are basically considered as the disappeared cells from the cell cycle. All transition and branching processes are time variant and heterogeneous. In addition, the regulatory mechanisms behind the decision for these transition or branching processes are random in nature. The model also predicts a high degree of variability in the molecular compositions and the time required to complete the cycle. Computational analysis of these three classes of cells is presented in the following subsections.

\subsection{Proliferation process}

The importance of the cell growth and proliferation are accelerated

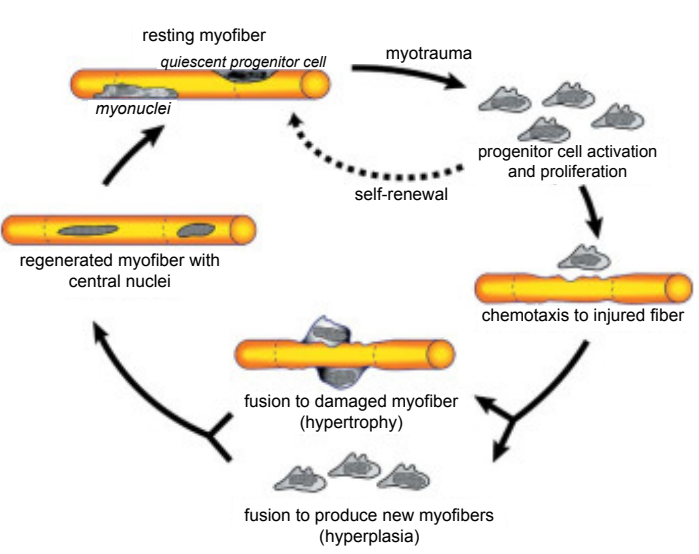

Figure 1: Myosatellite cell-life cycle [6]. 
not only for its crucial role in tissue (muscle) therapeutic process upon acute injury but also its ability to overuse. In fact, these are the most unique molecular properties of tissue or cell biology. This process is dependent on time as well as the quantity of the initial cell populations and their proliferation-able capabilities. The cellular growth rate is also a function of different other variables [13] including age, environmental factors and oxygen concentrations.

Now, consider $\mathrm{X}(t)$, if it represents total number of proliferationable cell populations at time t; then standard experimental expression for cell division (or proliferative cell growth) $\mathrm{X}(\mathrm{t})$ can be presented:

$\mathrm{X}(t)=2 \mathrm{X}(t-1) \delta(\gamma)-[\mathrm{D}(t)+\mathrm{Q}(t)] \gamma(t)$

where,

$t=1,2 \ldots \mathrm{T} ; \mathrm{X}(t) \approx 0$ for large value of $\mathrm{T}$ and

$X(0)=$ initial cell populations

$D(t)=$ differentiated cells

$Q(t)=$ disappearance or dead cells rocess

$\delta_{(y \max )}=0$ and usually occur for large value of $\mathrm{T}$.

Furthermore, when biological factor $\gamma$ has no influence on the cell division process then the contribution due to the second part of right hand side is negligible and vice versa.

Estimate of $\mathrm{X}(\mathrm{t})$ at time $\mathrm{t}=\mathrm{T}$ can also be formulated:

$(\mathrm{t})=2^{\mathrm{T}-1} \mathrm{X}(0)+\eta(t)$

such that,

$X(t)-(t)=0$

where $\eta$ is the effect on estimation process due to the molecular heterogeneity or biological factors; and $2^{(\mathrm{T}-1)}$ is the exponential doubling factor.

\subsection{Differentiation process}

In the cellular biology, cessation of proliferation capability can be termed as differentiation. Basically, it is a process through which a genetic cell transforms into a tissue specific cell in response to a specific intra and extra cellular signal. Myogenic cells are able to differentiate followed by fused to existing muscle fibers or form new fibers. This myogenic differentiation process is closely attached with the basic helix-loop-helix (bHLH) transcription factors [1,2]. On the other hand, myogenic regulatory factors (MRF4) appeared to function in acceleration or activation of muscle differentiation. Furthermore, differentiation process can be classified as a partial differentiation $\left(D_{\mathrm{p}}\right)$, which has the potential to become a proliferation-able cell; and as a terminally differentiation (D) or end cell, which is essentially an irreversible process. The relationship between proliferation and differentiation processes for the muscle stem cells can be conceptualized from the schematic diagram presented in figure 2.

Now, terminally differentiation process $\mathrm{D}(t)$ at time $t$ can be stated:

$D(X, t)=(1-\alpha) X(t)+\eta(t)$

and

$\alpha=\lambda+\beta$

$$
\begin{aligned}
& 0 \leq \lambda \leq 1 \\
& 0 \leq \beta \leq 1 \\
& 0 \leq \lambda+\beta \leq 1 \\
& a_{\text {max }}=1 \text { and } \\
& a_{\text {min }}=0
\end{aligned}
$$

where $\lambda$ and $\beta$ are proportionality factors for differentiated and dead cells respectively and inversely proportional to time $t$. These factors are influenced by $\eta$ and also dependent on the number of cell populations at $\mathrm{t}$.

Cell death or disappearance process: Like growth and differentiation, cell disappearance or cell death $Q(X, t)$ is influenced by several factors including age, environment and heterogeneity in cell populations. Basically, this process is considered as a departure of cells from the cell life cycle due to apoptosis, narcosis or return to quiescence or stems stage [2]. However, according to the proposed model, this transition process is also considered as a cessation of cell proliferationable capability. Moreover, this transformation process is also a function of time $t$ and dependent on $\lambda$ and $\beta$ as stated in eq. (4). Importantly, equations (3) and (4) can also be used to characterize and quantify the disappearance process of the cell populations.

\subsection{Decay process}

According to the proposed computational model, total cessation of the cell proliferation-able property due to the cell differentiation and cell disappearance can be termed as decay process. This irreversible process is complementary of growth process and accelerated by the same molecular events as stated for the cell growth. For example, if the cell populations $\mathrm{X}(\mathrm{t})$ can be considered as the function of time $t$; then using eq. (2), X ( $\mathrm{t}$ ) can be restated [14]:

$\mathrm{X}(t)=\mathrm{X}(0) 2^{-\mathrm{b}(\mathrm{t}-1)}+\eta(\mathrm{t})$

where $\mathrm{t}=1,2 \ldots \mathrm{T}$.

Now, the rate of decay:

$$
\frac{d x(t)}{d t}=-b\{X(t)+\eta(t)\}+\frac{d \eta(t)}{d t}
$$

where $\mathrm{b}$ is a proportionality constant and (-) sign indicates that the slope of $X(t)$ against $t$ is essentially a negative quantity since, cell population decreases with time $t$; and the parameter $\eta(t)$ refers to the heterogeneity in decay process and has the same influence on cell populations as stated in eq. (2). Now, for some suitable values of $\eta(t)$ as well as for the homogeneous cell populations, the rate of decay stated in eq. (6) can be restated:

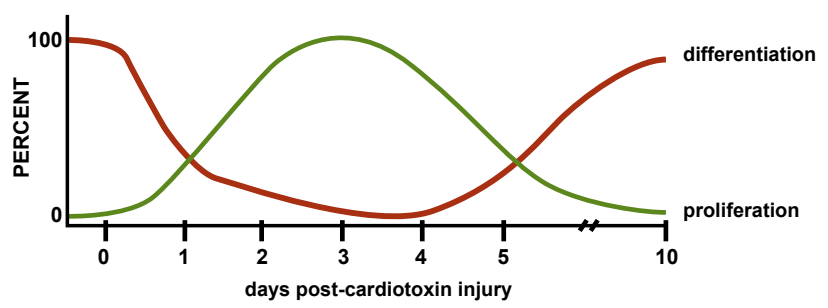

Figure 2: Muscle regeneration-proliferation and differentiation process [6] 


$$
\frac{d X(t)}{d t}=-b X(t)
$$

According to (7), it can be concluded that the rate of decay at particular time is proportional to the cell populations at that time. Therefore, from the outcomes of this equation, it is apparent that, cell populations at certain time $t$ can also be predictable.

\subsection{Stochastic cell theory}

The multi-branching stochastic models for the single stem cell populations in spatial-temporal domain can be presented as shown in figure 3 , however for the simplicity of this process, some branching parts are excluded from the figure $3[12,16,17]$. According to figure $3 a$, a proliferation-able stem cell is divided into two or one proliferation-able cell with the probability of $\mathrm{p}_{2}$ and $\mathrm{p}_{1}$ respectively. In the case of figure $3 \mathrm{~b}$, a proliferation-able stem cell is producing two or no proliferation-able cells with the probability of $p_{2}$ and $p_{0}$ respectively. In figure $3 c$, cell has the capability to produce one proliferative or two terminally differentiated cells with the probability of $p_{1}$ and po respectively. In figure $3 \mathrm{~d}$, one proliferationable cell is divided into two proliferation-able cells or one partially differentiated and one terminally differentiated cells or two terminally differentiated cells or one terminally differentiated and one dead cell with the probability of $\mathrm{p}_{2}, \mathrm{p}_{1}$ and po respectively. In figure $3 \mathrm{e}$, one proliferative cell is producing one proliferative cell and one partially differentiated cell or two terminally differentiated cells or one partially differentiated and one terminally differentiated cells with the probability of $p_{2}$, po and $p^{1}$ respectively. Finally, figure $3 f$ states that one proliferation-able cell can transform into a terminally differentiated cell without participating in any divisional process. The ability of a partially differentiated cell to become a proliferation-able cell is also apparent from figure $3 \mathrm{e}$. It is also worthy to mention that this branching process is homogeneous at a particular period of time and heterogeneous as a whole.

\subsection{In vitro cellular technology}

In this model, the proliferative capability of the cell population is characterized based on the in vitro experimental design, a design technology used in the laboratory for cell research instead of inside the body. Typically, growing new types of cells in the laboratory and transplanting them into the body for treating hereditary diseases is known as the cell therapy. The cell growing technology in the laboratory is known as the cell culture, where cells have the limited ability to exhibit their molecular properties due to the lack of permissive and repressive factors as compared to in vivo [1,2].

Cell culture: The observations of desired molecular markers under the influence of induced force are one of the main objectives of the cell culture. The overall observation period for the satellite proliferationable cell populations in the cell culture media usually takes 10-12 days [2]. Typically, this time period is also considered as a cessation time of the cells proliferative capabilities or the transformation time from the proliferation-able cell state to the terminally differentiated state. As the density of the cell increases with time, confluency of cell media is researched (i.e., when all of the potential cell growth areas are occupied). Confluence is highly unexpected, since at this stage cells have the limited capacity to grow and eventually lose their phenotypes with a higher possibility to die. To overcome this challenge, certain portion of the cell populations are removed frequently over the period of time from the primary culture media and re-plated into the new culture media, known as the subculture. Furthermore, the level of stimulation ability of the induced factors for the cell growing process is dependent on the

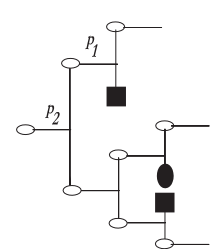

(a)

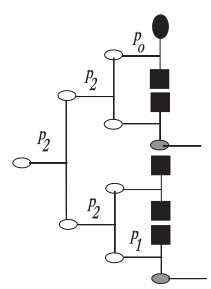

(d)

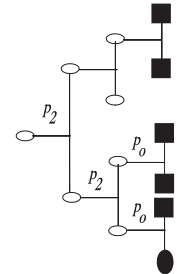

(b)

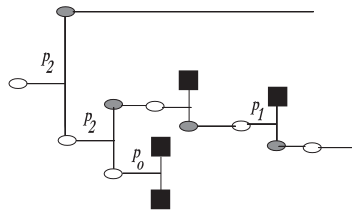

(e)

(c)
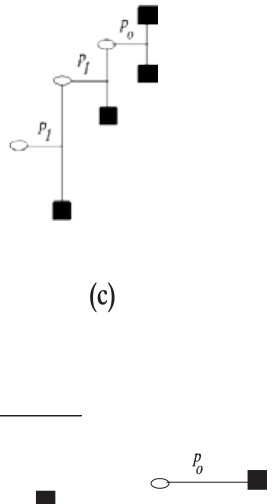

(f)

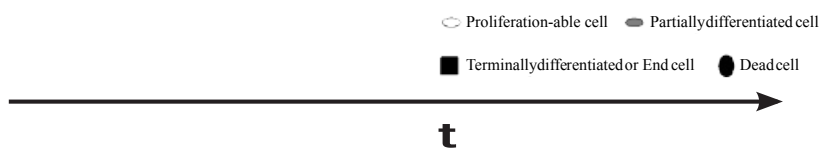

Figure 3: Stem cell branching model.

age and the cell proliferation-able capability of the cell populations in the culture media. Therefore, these two factors also play an important role for the cells to be a potential candidate for the cell culture.

Differences between in vitro and in vivo: Basically, in vitro is a dissociation of cells from the 3-dimensional geometrical environment (in vivo) to the 2-dimensional environment [2]. In the case of muscular stem cell, neonatal skeletal muscle is the main source of culture cell area for the in vitro laboratory experiment [1]. In most of the cases, cells in the in vitro lost their intra- and extracellular interactions because of the different aspects of the cell lines and the lack of heterogeneity. As a result, irregularity and inconsistency might occur for cells to spread out and express their activation and proliferation-able capabilities. On the other hand, lack of homeostatic regulatory factors and heterogeneity in the culture media could misinterpret the original stem for the overall cell populations. The characteristics of the cells in the in vitro are influenced by the induced force and environment factors including growth and hormones [2]. Furthermore, cells in the short term culture media may have the stability; however, heterogeneous cell property can produce variability in different points throughout the in vitro cell life cycle. Unlike in vivo, most of the cases, cultured cells are unable to exhibit their fundamental properties because of higher probability of cell contamination and tendency for rapid confluency; however, in vitro provides an enormous level of simplicity, where complexity of living organism is a great barrier for the clinical research.

\section{Computational Analysis}

Computational models play a vital role for experimentalists and biomedical researchers in recent year as their collective understanding increasingly elucidating the complicated intramolecular actions and tissue philosophy [15-19]. Dynamics of the proliferation-able muscle stem cells followed by myotrauma is a function of time and dependent on the growth factors as well as intrinsic and extrinsic molecular properties [1]. In this proposed model, cells are primarily classified 
based on their proliferation-able capabilities, since use of other molecular properties including morphology or phenotypes for their classifications would be a nontrivial process. The dynamic behaviours presented here are excited and accelerated by the activation signal and the heterogeneity in the cell populations. Typically, declination for the dynamic behaviour of growth process increases with the decrease of the cell proliferation-able probability. These properties are random in nature and the regulatory mechanisms of the proliferation- able cell populations are influenced by these properties. Execution and design architecture of the proposed model can be conceptualized, from the following subsections, the model diagram illustrated in figure 4 and proposed model stated by eq. (8).

\subsection{Assumptions}

Proposed convergent model is purely computational and based on the stochastic estimation theory. Moreover, stem cell populations under study exhibits homogeneous cell characteristics during their transition cycle and heterogeneous characteristics as a whole. In this context, stem cell is considered as a proliferation-able and participates in cell growth process as long as it has the capability to self-renewal or grows through the two most classical divisional processes known as symmetrical and asymmetrical cell divisions [8-12]. Furthermore, muscle stem cell with initial cell population 4 (number of initial cells) has been considered and studied over the time period 0-10 days. The main reason to consider myogenic satellite cell is that, these cell populations are initially (unperturbed state) non-proliferative and stay in the quiescent state $[1,6]$ and require an external stimuli (myotrauma) for their activation and proliferation [1]. The concept of these initialization and activation processes resemblance to the model initialization, execution, estimation and termination processes. In addition, computational aspects for this cell population are still undefined and very little is known regarding the molecular phenotype of the satellite cell [1]. Therefore, understanding the ability for myogenic cell to proliferate and differentiate could improve the capability to treat debilitating muscular diseases, like cachexia, cardiac and muscular dystrophy.

\subsection{Discrete-time computational model}

Quantitative understanding for the cell growth dynamics is the fundamental aspect of cell biology; since, it institutes the regulatory mechanisms responsible for the expansion of cell population and acceleration of their therapeutic process towards the appropriate lineage. On the other hand, sense of proliferation-able cell populations is the probability or tendency of cells to increase their volume. Therefore,

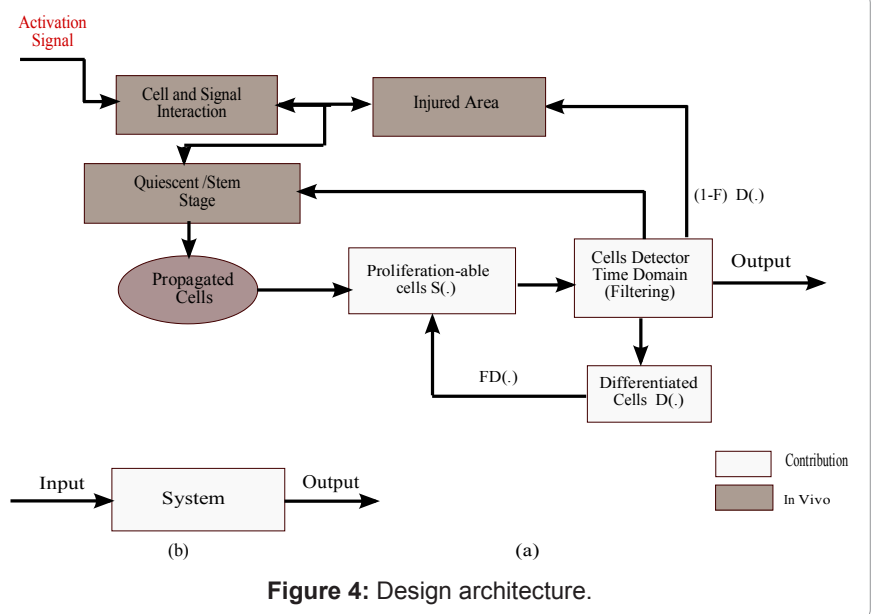

quantification of this cell population is an essential prerequisite to manipulate stem cell biology.

Furthermore, this discrete time stochastic model is compiled under the assumption that fundamental concept of the growth cycle of stem cell is the same, where they have the ability to undergo three stages, such as, proliferation, differentiation and death or disappearance. However, these stages can be influenced by the respective environmental or growth factors. In addition, according to this model, proliferative capability of the cell population is characterized based on the in-vitro experimental design, a design technology used in the laboratory for cell research instead of inside the body.

Now, assume that proliferation process is stochastic in nature with probability $\mathrm{R}$, where $\mathrm{R}$ is a discrete random variable with probability distribution $\mathrm{P}_{\mathrm{R}}=$ pi $\{\mathrm{i}=0,1,2\}$; and $\mathrm{S}(0), \mathrm{D}($.$) and \mathrm{Q}($.$) are$ the initial cells, differentiated cells and disappearance of cells (death or apoptosis) respectively. In this context, proliferation means ability of cell populations to divide through symmetrical (two cells of same cellular fates or progeny) or asymmetrical (two cells of different cellular fates or progeny) cell division as shown in figure $5[7,16]$. Furthermore, if parameter $\mathrm{F}$ is the fraction of differentiated cell populations, which turned into the proliferation-able state during certain time cycle t. The model equation for the proliferation-able cell populations in discrete time domain at time $t$ can be formulated:

$\mathrm{S}(t)=\mathrm{RS}(t-1)+(2-R)[(F-1) D(S, t-1)-Q(S, t-1)](-1)^{(\mathrm{R}-1)}$

where,

$t=1,2,3 \ldots \mathrm{T}$ (usually considered no of days);

$\mathrm{S}(t) \approx 0$ for large value of $T$;

$\mathrm{F}$ is an arbitrary variable, with $0 \leq F(t) \leq 1$.

Furthermore, probability distribution of discrete random variable $\mathrm{R}(0 \leq \mathrm{R} \leq 2)$ :

$$
P_{R}(R)=\left\{\begin{array}{lll}
p_{2} & \text { if } \mathrm{R}=2 \\
p_{1} & \text { if } \mathrm{R}=1 & p_{2}+p_{1}+p_{0} \\
p_{0} & \text { if } \mathrm{R}=0
\end{array}\right.
$$

\subsection{Convergence properties of discrete time model}

Convergence approach presented here is purely computational. The basic concept of this methodology is to show that, the model equation stated in eq. (8) represents the cell proliferation process and cessation of proliferation capability occurs during their finite life cycle $\mathrm{T}$. In other words, the difference between eq. (1) and (8), $\mid \mathrm{S}$-X $\mid$, converges to zero for finite value of $T$ and $S \rightarrow X \rightarrow 0$ at the end of the growth cycle, where $X$ and $\mathrm{S}$ represent proliferation-able cells in the case of clinical experiment and computational model respectively.

Moreover, parameters in eq. (8) are function of time and dependent on the proliferation ability of the cell population; and accordingly, it is assumed that all cells execute inherent homogeneous characteristic during each transition cycle, however their property is inherently heterogeneous as a whole.

Now, according to the probability distribution and underlying assumptions including homogeneous and heterogeneous cell characteristics, consider that all cells would experience one of the following three possible proliferation properties during transition cycle from $t$ to $t+1$ :

Property $1: \mathrm{P}_{\mathrm{R}}=\mathrm{p}_{2}$

In this case, each cell with $\mathrm{R}=2$ has the capability to divide only 


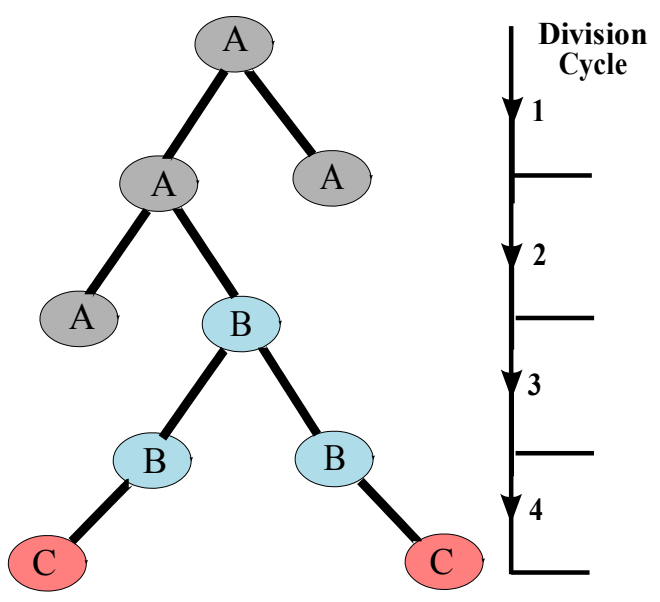

Symmetric and Asymmetric Cell Divisions

Figure 5: Single cell division process [7]

into two progenitor cells through symmetric or asymmetric process. There is no differentiated or dead (or disappearance) cell occurred at this transition cycle with this probability distribution. Under these assumptions, regardless of the values of other model parameters, the outcome of discrete time model eq. (8) represents proliferative cell dynamics, which can be stated as:

$$
\mathrm{S}(t)=2 S(t-1)
$$

\section{Property 2: $\mathrm{P}_{\mathrm{R}}=\mathrm{p}$}

According to this distribution property, stem cell has the limited proliferation-able capability with $\mathrm{R}=1$ and produces one progenitor cell and one differentiated cell or end cell. Outcome of the model with this probability distribution, underlying assumptions and $\mathrm{F}=0$ :

$$
\mathrm{S}(t)=\mathrm{S}(t-1)-\mathrm{D}(S, t-1)-\mathrm{Q}(S, t-1)
$$

Property 3: $\mathrm{P}_{\mathrm{R}}=\mathrm{p}_{0}$

In this stage, stem cell lost its proliferative capability with $R=0$ through differentiation or disappearance process. However, some differentiated cells have the capability to become proliferationable (i.e, $\mathrm{F} !=0$ ) cells and start the cell division process until $F=0$. But, based on the biological hypothesis, $F=0$, can be considered when the value of $T$ is large. Hence, the model stated in (8) with this property represents the cessation of cell proliferation-able capability, which is also known as the end of cell proliferation life cycle, and can be stated:

$$
S(t)=2[D(S, t-1)-Q(S, t-1)] \rightarrow 0
$$

in nonproliferation-able sense.

According to the three stated properties based on probability distribution, cessation of proliferation or growth process occurred when cells exhibit property 3 , and while property 1 is essentially representing the growth only process for proliferation-able cell populations. On the other hand, in the in vitro experimental observations, it has been found that cessation of cell proliferation capability of cell populations occurred at the end of the cell proliferative life cycle $T$, where $T$ is very large and finite. Therefore, the model would converge with experimental outcome once transition cycle executes distribution property 3 for large value of $\mathrm{T}$.

\section{Computationally,}

$$
|\mathrm{S}-\mathrm{X}| 0
$$

Therefore,

\section{$\mathrm{S}(t) \quad$ Converge, subject to: $R=0$ for large $T$}

Furthermore, it is also apparent from the aforesaid analysis that model stated in eq. (8) converges with eq. (1); and discrete random variable $R$ and model parameter $F$ are the definitions for the biological parameters $\gamma$ and $\eta$ under the assumption that model (cell cycle) would execute property 3 for large finite value of $T$. More importantly, like clinical hypothesis, it is also apparent from the above analysis that homogeneity and heterogeneity induced by the stated properties are also the necessary characteristics for the proposed model in order to maintain the continuation and controlling of the overall cell dynamics within the cell populations.

\section{Model Estimation using Kalman Filter}

Now, consider an unknown dynamic system with a state vector S (t) and system is driven by a random noise. Here, model stated by eq. (8) represents state equation, since it characterized the computational process of proliferation state. Using the properties of the discrete-time domain filter (Kalman Filter) [18-20], the state equation for transition from $S(t)$ to $S(t+1)$ state can be written as:

$$
\mathrm{S}(t+1)=\mathrm{C}(t) \mathrm{RS}(\mathrm{t})+(2-\mathrm{R})[(F-1) D(\mathrm{~S}, t)-Q(\mathrm{~S}, t)](-1)^{\mathrm{R}-1}+\mathrm{v}(t)
$$

Where $C(t+1 \mid t)$ and $v(t)$ are the state transition matrix and processed noise respectively. This noise is assumed to be white Gaussian and zero mean; and the covariance of the process noise is assumed $\Gamma=\Psi_{p}^{2} I \quad$ where $I=1$. On the other hand, for a stationary system, the state vector $S(t)$ would be fixed, and the transition matrix $C(t+1 \mid t)$ can be considered as identity matrix, but for the proposed nonstationary system, a more complex form of $\mathrm{C}(\mathrm{t}+1 \mid \mathrm{t})$ need to be developed, so that computational model can track the changes in response to the change of the environment [21-26].

Now, the measurement equation (desired) for the state at $t+1$ can be stated as:

$$
Z(t+1)=H^{T}(t+1) S(t+1)+\rho(t+1)=H[t, S]+\rho(t+1)
$$

where $\rho(t+1)$ is white Gaussian measurement noise with zero mean and covariance is given as [18-20]:

$$
\mathrm{E}[\rho(t) \rho(i)]=\sigma_{m}^{2}(t) \delta_{t i}
$$

where $(.)^{\mathrm{T}}$ represents transpose.

Estimate of measurement (desired value) $\mathrm{Z}(t+1)$ can be stated as:

$$
\hat{Z}(t+1 \mid t)=H^{T}(t+1) \hat{S}(t+1 \mid t)
$$

Mean square error (MSE) between desired and computational outputs:

$$
e(t)=Z(t+1)-(t+1) \max _{[\mathrm{D}, \mathrm{Q}]} M S E=\mathrm{E}\left[\mathrm{e}^{2}(\mathrm{t})\right]_{\min }
$$

The objective is to find out the minimum value of MSE in such a way that model converges for finite large value of $T$, where $t=0,1, \ldots \ldots$, $T$. More importantly, using the approximation of cell biology and the illustrated properties in section 3.2, model stated by eq. (8) would also be converged for large values (population) of $\mathrm{D}(t)$ and $\mathrm{Q}(t)$ in MSE sense. Since at this stage, proliferation property has a negligible effect on the growth process due to the influence of non-proliferative cell populations. Furthermore, according to clinical theory, this 


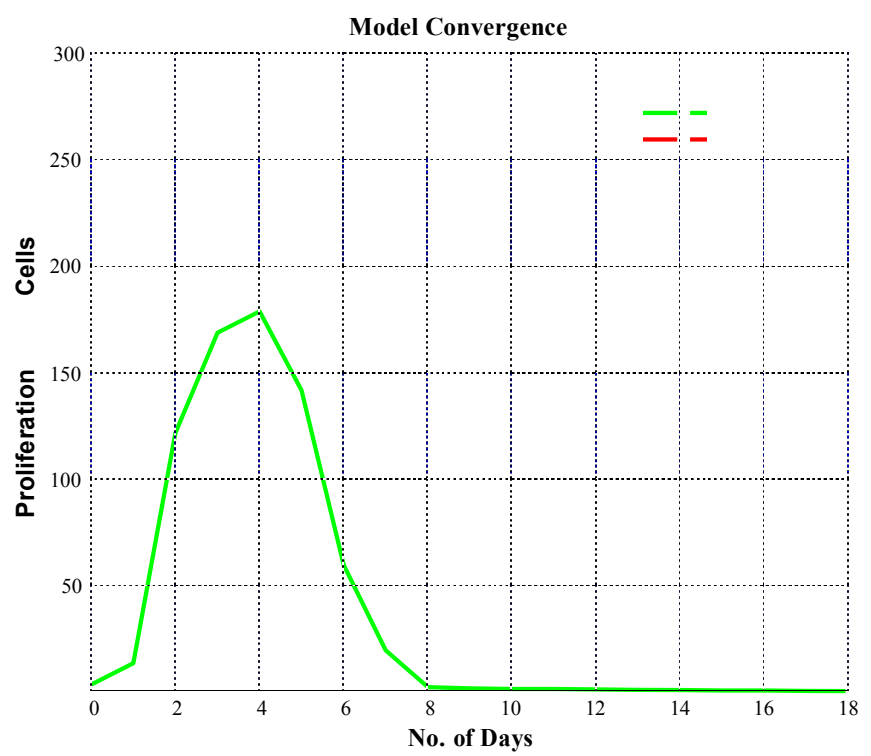

Figure 6: Model convergence.

approximation is also observable at the end of cell proliferative life cycle, where the value of $\mathrm{T}$ is large and finite.

Now, innovation of measurement residue $[18,20]$ :

$v(t+1)=Z(t+1)-\hat{Z}(t+1 \mid t)$

State prediction is:

$$
\hat{\mathrm{S}}(t+1 \mid t)=C(t) \hat{\mathrm{S}}(t \mid t)
$$

where,

$\hat{\mathrm{S}}(t \mid t)=\mathrm{E}[\mathrm{S}(t) \mid Z(t)]$

Hence, state update equation can be stated as:

$\hat{\mathrm{S}}(t+1 \mid t+1)=\hat{\mathrm{S}}(t+1 \mid t)+\mathrm{k}(t+1) v(t+1)$,

where $\mathrm{k}(\mathrm{t})$ is filter gain and can be stated as:

$$
k(t+1)=P(t+1) \mid t) H^{T}(t+1) M(t+1)-1
$$

and estimated (predicted) state conditional covariance [18]:

$\mathrm{P}(t+1 \mid t)=C(t) P(t \mid t) C T(t)+\Gamma(t)$

Innovation covariance:

$$
\mathrm{M}(t+1)=\sigma_{m}^{2}(t+1)+H^{T}(t+1) \cdot P(t+1 \mid t) H(t+1)
$$

Covariance update:

$$
\mathrm{P}(\mathrm{t}+1 \mid \mathrm{t}+1)=\mathrm{P}(\mathrm{t}+1 \mid \mathrm{t})-\mathrm{k}(\mathrm{t}+1) \mathrm{M}(\mathrm{t}+1) \mathrm{k}^{\mathrm{T}}(\mathrm{t}+1)
$$

Alternate (using Matrix Lemma) form of covariance update [18,27]:

$$
P(t+1 \mid t+1)-1=P(t+1 \mid t)-1+H^{T}(t+1)\left[\sigma_{m}^{2}\right]-1 H(t+1)
$$

For large initial value condition, if first term of right hand side of eq. (25) can be neglected, then eq. (25) can be restated as:

$$
P(t+1 \mid t+1)^{-1}=H^{T}(t+1)\left[\sigma_{m}^{2}\right]^{-1} H(t+1)
$$

This is a recursive and linear process starting from eq. (14), and the process would stop once the stop criteria are met. Furthermore, difference illustrated in eq. (17) represents the differences between the desired and the computational outputs estimated using the proposed model in eq. (8) with underlying assumptions. If the desired output $Z$ $(t)$ can be interpreted as an approximation of the actual desired output using the underlying assumptions, then the actual desired output $A_{d}$ $(t)$ and its approximation $\mathrm{Z}(t)$ with approximation error $\mathrm{E}_{\text {appr }}(t)$ can be related as:

$$
\mathrm{A}_{\mathrm{d}}(t)=\mathrm{Z}(\mathrm{t})+\mathrm{E}_{\text {appr }}(t)
$$

If $\mathrm{A}_{\mathrm{d}}(t)$ and $\mathrm{E}_{\text {appr }}(t)$ are considered as white Gaussian, zero mean and uncorrelated, then

$$
\sigma(\mathrm{t})^{2}=M M S E+\mathrm{E}\left[\mathrm{E}_{\text {appr }}\right]
$$

Therefore, if the measurement $\mathrm{Z}(t)$ is generated using the realistic approximation of the actual out-comes then $\sigma^{2}(t)$ would be approximated based on the MMSE that can be achieved by the underlying assumptions and the filtering model stated above. For non stationary environment, the state transition matrix $\mathrm{F}(t+1 \mid t) \neq 0$ and $\Psi_{p}^{2} \neq 0$ will make the state equation unstable. If the state covariance matrix $\Gamma$ represents the total uncertainty due to the adapting stationary environment assumption represented by using identity state transition matrix in eq. (13) and eq. (22). The effect associated with this deviation due to the nonstationary environment prevents Kalman filter gain from decaying to the values that are too small. Therefore, estimation of $\hat{\mathrm{S}}$ must be able to follow the variation due to the non stationary heterogeneous environment.

\section{Simulations}

The outcome and the performance of the proposed discrete computational model presented here, in figures 6 and 7 , are based on the synthetic data and over the time period 0-10 days $(T=10)$ (normalized and scaled). Furthermore, time domain filter, Kalman filter, is used in order to analyze the efficiency of the proposed computational architecture, and found that the trajectory from the estimated outcome is the close approximation of the synthetic truth.

Figure 6 shows proliferation-able cell cycle in temporal domain, and found that the model has converged for large value of $t(=T)$. This computational simulation is performed over 0-10 days with initial cell population at day zero is 4 . According to this figure, upon receiving the excitation pulse (i.e., execution of model), quiescent (initial) cells become proliferation-able cells and start to grow. It also shows that cells proliferate extensively within 2-4 days, and gradually start to lose their propagation property after the day 4 , and complete cessation of this process occurs within 7-8 days. The cessation of proliferation-able property for this model is slightly higher than experimental expectation (i.e., typically 5-6 days), because of the property of the induced model parameter F. Since, based on this property, some of the differentiated cells have the ability to be proliferation-able cells and start to participate in the division process.

Figure 7a represents the comparison between time domain filter outcome (estimated) and the model truth. According to this figure, estimated proliferating process is close to the synthetic truth from day $0-2$, but slightly deviated from the truth from day $2-4$ due to the influence of extensive (i.e., model parameters $\mathrm{R}$ and $\mathrm{F}$ or doubling factor) growth of proliferation-able cell populations. On the other hand, de-proliferation process is close to the truth data after day 4 until day 6 , but it is slightly deviated from the truth after day 6 due to the de-differentiated parameter $\mathrm{F}$, however for large value of $\mathrm{T}(=10)$, it converged towards the synthetic truth. More importantly, mean square error (MSE) presented in figure $7 \mathrm{~b}$ for this computational process is below 1 for most of the cases after 20 iterations.

Figure 8 states the comparison among the distribution properties 


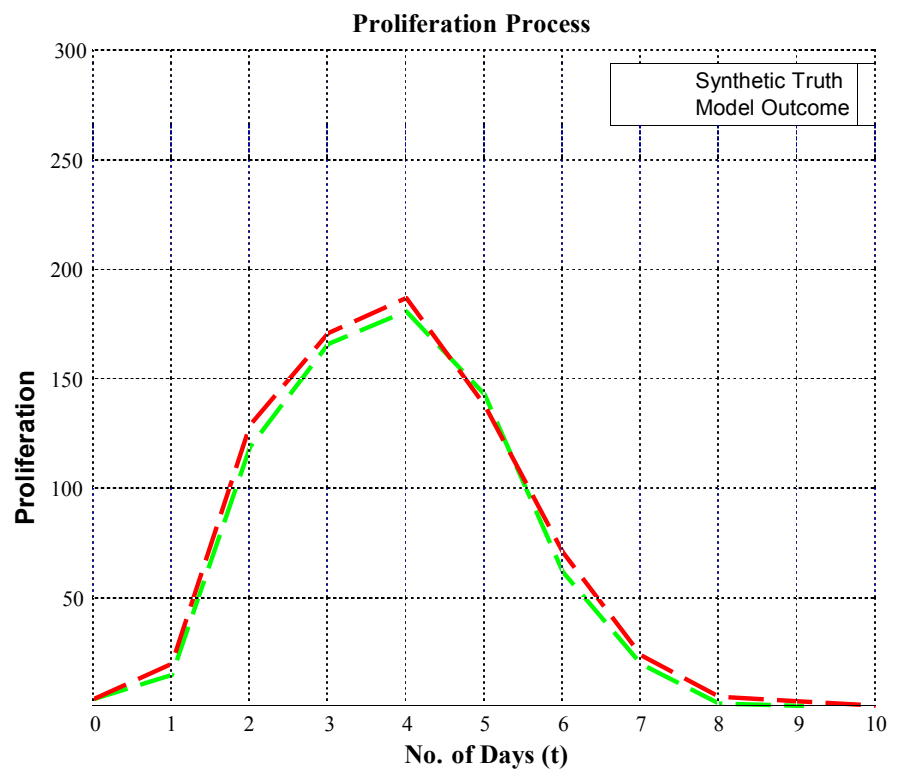

(a) Proliferation Process

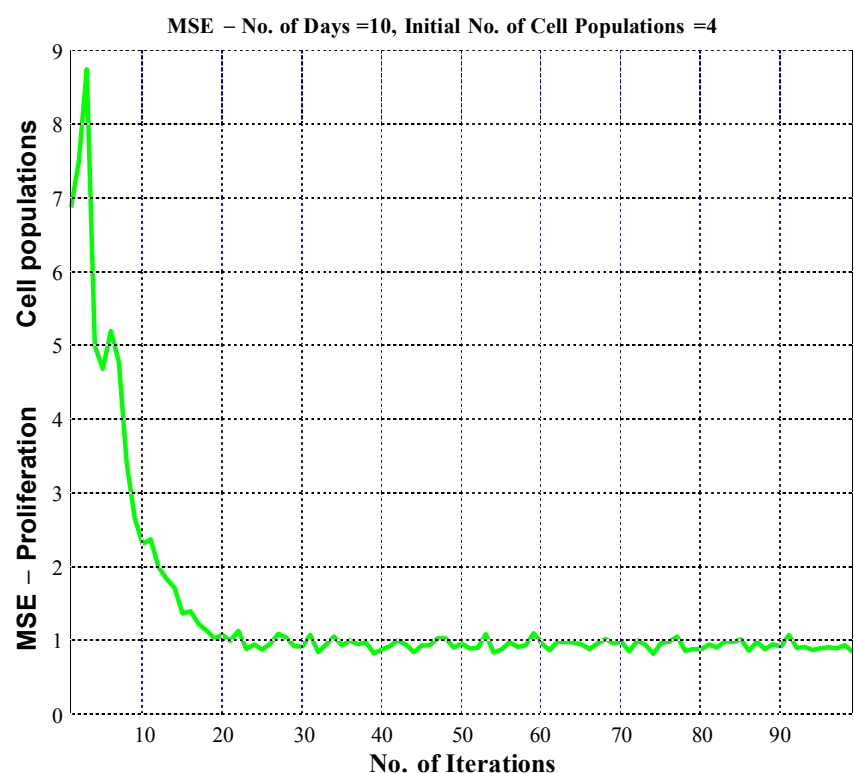

(b) MSE-proliferation-able cell populations

Figure 7: Computational model (simulated output) for stem cells.

stated in section 3.2, over the time period 0-10 days. Distribution property represented by property 1 is a (homogeneous) growth only process, while property 2 represents the state where cell has the equal probability to execute proliferation and differentiation processes. Furthermore, property 3 is simply a decay process. The performance of the proposed model and its combined effect on the computation of the proliferation process, and its convergence property are apparent from this simulation; and figure $8 \mathrm{~b}$ is a snapshot of figure $8 \mathrm{a}$ for better understanding of the overall estimation process.

\section{Conclusions}

In this article, a simplest nontrivial discrete time convergence theory for proliferation-able stem cells and its estimation using Kalman filter (KF) has been proposed. Furthermore, muscle stem cell with initial cell population 4 has been considered and studied over the time period 0-10 days. On the other hand, the design approach of this model is purely computational, and the fundamental concept is to analyze the quantitative aspect of proliferation-able stem cells and compare it with the experimental hypothesis. This analysis is based on the observations, predictions. In this architecture, linear time variant filtering technique has been used, where homogeneous cell populations for a particular transition period and heterogeneous cell populations as a whole have been considered.

\section{Discussions}

Stem cell populations are in quiescent, but upon receiving the stimuli they become activated and undergo proliferation, differentiation and disappearance processes. Growth of these cell populations is random in nature and it is extremely influenced by the number of initial cells and their proliferative capacities. The properties in section 3.2 state that proposed hypothesis converges when model exhibits property 3 (i.e., $R=0$ and $F=0$ ) within its finite transition period $T$. According to the experimental observations, this property is achievable for large value of $T$, where cells completely lost their proliferation capability (Figure 2). Therefore, proposed computational architecture is a close approximation under the assumption that model would reach at the stage, where probability distribution for random variable $R=0$ and de-differentiation parameter $F=0$ for large finite value of $T$. Furthermore, like experimental hypothesis, it is apparent that model executes heterogeneous and homogeneous characteristics because of the coordinated induced properties stated in section 3.2. In fact, like clinical biology, these properties are also the necessary conditions in order to control the cell dynamics for genetic stability of the model.

Furthermore, the objective of section 4 is to evaluate the performance of proposed model using Kalman filter. This bioengineering system is essentially linear, and linear dynamics of this filter has shown that model converges with the expected output in MSE sense. According to trajectories in section 5, it is found that proposed output is slightly deviated from the truth, and the main reason for this deviation is due to the de-differentiation parameter F. This induced parameter has also influenced the cessation of proliferation period, and as a result it is also observable that this cessation time is higher than the experimental expectation.

\section{Applications}

Typically, proliferation is the growth process which increases the cell volume in the cell culture or inside the body. Studies found that, regulatory mechanism of stem cell growth cycle can be characterized by the factors, responsible for this process $[1,2]$. However, unlike in-vivo, studies of growth process in the laboratory faces challenges due to the, genetic instability, variability and cell contamination in the one hand; and lack of permissive and repressive factors responsible for cellular activities on the other hand [1,2]. As a result, an extensive number of in vitro experiments need to be run in the laboratory in order to optimize the clinical understanding. But, like in vivo, in vitro studies are 


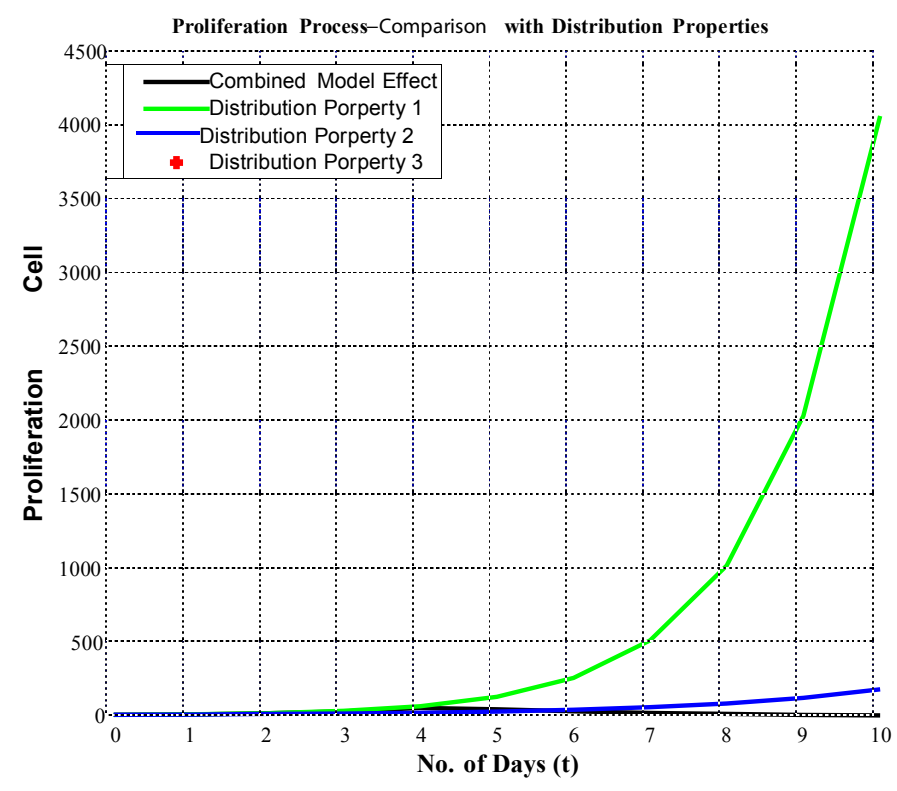

(a) Time period 0-10 days

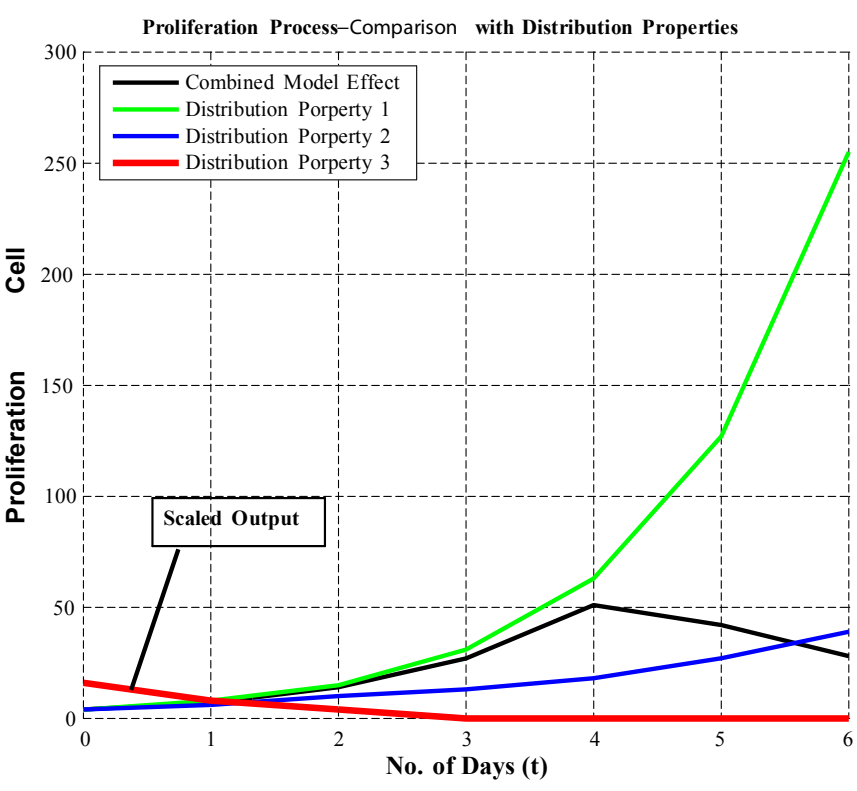

(b) Time period 0-6 days

Figure 8: Proliferation process-comparison of distribution properties.

also facing ethical, financial and resource limitations in time domain. Hence, proposed estimation model could be used as a bioexperimental tool to study the possible pattern of the stem cells growth process in the laboratory (i.e., in vitro environment).

Therefore, the potential application of this model is in the biological research laboratory as an estimated experimental tool in order to predict and estimate a biological process. For example, consider an experiment, which is conducted in the laboratory using a biological system represented by figure $4 \mathrm{~b}$; when input is a biological raw input and output is an experimental outcome. The biological transfer function (system) which is responsible to produce this end result is essentially a composite biological culture media (system) induced by the experimentalists under a specific clinical environment. In fact, this composite biological system is the main area of interest for the experimentalists; since, the sample of this system characterizes the nature of the induced biological regulatory factors required for this process. Now, replace this known biological system in figure $4 \mathrm{~b}$ with the model stated in eq. (8) and represented by figure $4 \mathrm{a}$, and run the estimation process in time domain. As the input and the expected output are known from the previous biological experiment for this estimation system, it can be stated from the final expected outcome of the model that the characteristics of the system of the proposed model resembles to that biological system. Besides, model parameters can also be comparable with the induced biological factors under that environment. As a result, this estimated outcome can be used as a predicted model for the next laboratory experiments under the similar environment. Therefore, this model would be useful to enhance and simplify the decision making ability for the researchers by predicting the characteristics of the biological factors based on the multi-dimensional interests before preparing and executing the laboratory experiments. Execution of repeated experiments in laboratory could be very complex and infeasible otherwise.

More importantly, model is based on the synthetic data, and as a consequence, some unavoidable computational irregularity and inconsistency may occur due to the lack of coordination between the biological dynamics and the underlying stochastic distribution of the engineering hypothesis. Finally, the proposed quantitative analysis of proliferation-able stem cells stated in eq. (8) may also be implementable for differential cell populations; however, unlike proliferative cell, this process is slow and stayed in that state for a long period of time. In conclusion, this computational analysis is based on the observable biological cell properties for both of the cell populations in the laboratory, from engineering point of interests; and due to limited excess to the biological resources, perhaps it would be a challenging task to summarize the model performance under realistic experimental framework without having more extensive validation with the clinical hypothesis.

\section{Acknowledgements}

The authors thank Dr. Thomas J. Hawke (McMaster University) and Dr. Rennian Wang (University of Western Ontario) for their extraordinary advises and biomedical resources; and are also grateful to Rabita A. Alamgir (University of Virginia) for her exceptional assistance throughout this research.

\section{References}

1. Hawke TJ, Garry DJ (2001) Myogenic satellite cells: physiology to molecular biology. J Appl Physiol 91: 534-551.

2. Freshney R (1987) Culture of Animal Cells - A Manual of Basic Technique. Alan $\mathrm{R}$ Liss Inc, New York.

3. Palsson BO, Bhatia SN (2009) Tissue Engineering. Pearson Education Inc.

4. Cornelison DD, Wold BJ (1997) Single-cell analysis of regulatory gene expression in quiescent and activated mouse skeletal muscle satellite cells. Dev Biol 191: 270-283.

5. Snow MH (1977) The effects of aging on satellite cells in skeletal muscles of mice and rats. Cell Tissue Res 185: 399-408.

6. (2007) Satellite Cells, the Primary Stem Cells of Adult Skeletal Muscle. Partia Immortalization.

7. The National Institutes of Health Resource for Stem Cell Research (2011) Stem Cell Information. 
Citation: Malek O, Venetsanopoulos A, Alamgir L, Alirezaie J, Krishnan S (2013) A Discrete-Time Convergence Model for Proliferation-able Stem Cell and its Estimation using Kalman Filter. J Bioengineer \& Biomedical Sci 3: 119. doi:10.4172/2155-9538.1000119

8. Baker M (2009) State of the Stem Cells. Nature Reports Stem Cells.

9. Relaix F, Marcelle C (2009) Muscle Stem Cells. Current Opinion in Cell Biology 21: $748-753$

10. Roeder I, Lorenz R (2006) Asymmetry of stem cell fate and the potential impact of the niche: observations, simulations, and interpretations. Stem Cell Rev 2: $171-180$

11. Tomasetti C, Levy D (2010) Role of symmetric and asymmetric division of stem cells in developing drug resistance. Proc Natl Acad Sci U S A 107: 1676616771.

12. http://en.wikipedia.org/wiki/Stem-cell

13. Pyaskovskaya ON, Kolesnik DL, Kolobov AV, Vovyanko SI, Solyanik GI (2008) Analysis of growth kinetics and proliferative heterogeneity of Lewis lung carcinoma cells growing as unfed culture. Exp Oncol 30: 269-275.

14. Aitken MRF, Aitken M, Broadhurs B, Hladky SB (2010) Mathematics for Biological Scientists. Garland Science.

15. G. Vries, "What is Mathematical Modeling", Department of Mathematical Sciences, University of Alberta.

16. Nance RE, Rajasimha HK, Samuels DC (2004) A Simulation Methodology in Modeling Cell Divisions with Stochastic Effects. Proceedings of the Winter Simulation Conference.

17. Ro S, Rannala B (2001) Methylation patterns and mathematical models reveal dynamics of stem cell turnover in the human colon. Proc Natl Acad Sci U S A 98: 10519-10521.
18. Bar-Shalom Y, Li X, Kirubarajan T (2001) Estimation with Applications to Tracking and Navigation. CR YBS, Artech House, Norwood, MA.

19. Fuss H, Dubitzky W, Downes CS, Kurth MJ (2005) Mathematical models of cell cycle regulation. Brief Bioinform 6: 163-177.

20. Malek O, Venetsanopoulos A, Anpalagan A (2010) A Comparison Study between Wiener and Adaptive State Estimation (STAP-ASE) Algorithms for Space Time Adaptive Radar Processing. Proceedings SPIE: Mathematics of Data/Image Coding, Compression, and Encryption with applications XII 7799.

21. Piotrowska MJ, Enderling H, an der Heiden U, Mackey M (2008) Mathematical Modeling of Stem Cells Related to Cancer. Nova Sceince Publishers.

22. Yakovlev A, Yanev N (2006) Branching stochastic processes with immigration in analysis of renewing cell populations. Math Biosci 203: 37-63.

23. Bjerknes M (1986) A test of the stochastic theory of stem cell differentiation. Biophys J 49: 1223-1227.

24. Feldman JL, Stockdale FE (1992) Temporal appearance of satellite cells during myogenesis. Dev Biol 153: 217-226.

25. Ganusov VV, Pilyugin SS, de Boer RJ, Murali-Krishna K, Ahmed R, et al. (2005) Quantifying cell turnover using CFSE data. J Immunol Methods 298: 183-200.

26. Furusawa C, Kaneko K (2001) Theory of robustness of irreversible differentiation in a stem cell system: chaos hypothesis. J Theor Biol 209: 395-416.

27. Simon D, Chia TL (2002) Kalman Filtering with State Equality Constraint. IEEE Transactions on Aerospace and Electronic Systems 3: 128-136. 